\title{
First reported use of real-time intraoperative computed tomography angiography image registration using the Machine-vision Image Guided Surgery system: illustrative case
}

\author{
*Harsh Wadhwa, BS, Karen Malacon, BA, Zachary A. Medress, MD, Christopher Leung, BS, Matthew Sklar, BS, and \\ Corinna C. Zygourakis, MD
}

Department of Neurological Surgery, Stanford University, Palo Alto, California

\begin{abstract}
BACKGROUND Vertebral artery injury is a devastating potential complication of C1-2 posterior fusion. Intraoperative navigation can reduce the risk of neurovascular complications and improve screw placement accuracy. However, the use of intraoperative computed tomography (CT) increases radiation exposure and operative time, and it is unable to image vascular structures. The Machine-vision Image Guided Surgery (MvIGS) system uses optical topographic imaging and machine vision software to rapidly register using preoperative imaging. The authors presented the first report of intraoperative navigation with MvIGS registered using a preoperative CT angiogram (CTA) during C1-2 posterior fusion.
\end{abstract}

OBSERVATIONS MvIGS can register in seconds, minimizing operative time with no additional radiation exposure. Furthermore, surgeons can better adjust for abnormal vertebral artery anatomy and increase procedure safety.

LESSONS CTA-guided navigation generated a three-dimensional reconstruction of cervical spine anatomy that assisted surgeons during the procedure. Although further study is needed, the use of intraoperative MvIGS may reduce the risk of vertebral artery injury during C1-2 posterior fusion.

https://thejns.org/doi/abs/10.3171/CASE2125

KEYWORDS machine vision; navigation; atlantoaxial; cervical fusion; CTA

C1-2 fusion is a challenging procedure because of complex neurovascular anatomy that increases surgical risks and necessitates highly accurate screw positioning. Although a rare occurrence, vertebral artery injury during cervical spine surgery can cause catastrophic bleeding, permanent neurological impairment, stroke, and/ or death. ${ }^{1,2}$ Reported complication rates vary based on surgical approach, with $\mathrm{C} 1-2$ posterior fusion carrying the highest risk of vertebral artery injury, ranging from $4.1 \%$ to $8.2 \%$ of cases. ${ }^{3-6}$ Advanced imaging is an essential part of preoperative assessment, and a computed tomography angiogram (CTA) is often obtained preoperatively to carefully delineate vascular anatomy and provide guidance on safe screw placement. Use of intraoperative fluoroscopy or computer-assisted navigation during surgery may further reduce surgical complication risk and improve screw accuracy. ${ }^{7,8}$

The Machine-vision Image Guided Surgery (MvlGS) system (7D Surgical, Inc.) is an intraoperative spinal navigation system that uses optical topographic imaging. Unlike other navigation systems that rely on intraoperative fluoroscopy or CT scans, MvIGS uses nonionizing structured light to obtain a three-dimensional (3D) surface scan of patient anatomy that is automatically registered to the patient's preoperative CT scan within seconds. ${ }^{9,10}$ This technology was initially developed to improve surgical workflow and allow intraoperative CT-guided navigation without the need for intraoperative scanning time and without intraoperative surgeon, staff, and patient radiation exposure. However, as this technology is used in more spinal procedures, additional advantages of MvIGS become apparent, such as the ability to navigate intraoperatively with a CTA instead of a standard CT scan. This advantage allows simultaneous visualization of bony and vascular anatomy and the critical spatial relationships between these structures. It also enables visualization of the extraosseous course of the vertebral artery, which is particularly useful in posterior $\mathrm{C} 1-2$ fusion procedures.

ABBREVIATIONS 3D = three dimensional; CT = computed tomography; CTA = computed tomography angiogram; MvlGS = Machine-vision Image Guided Surgery. INCLUDE WHEN CITING Published May 3, 2021; DOI: 10.3171/CASE2125.

SUBMITTED January 11, 2021. ACCEPTED February 11, 2021.

$*$ H.W. and K.M. contributed equally to this work.

(c) 2021 The authors, CC BY-NC-ND 4.0 (http://creativecommons.org/licenses/by-nc-nd/4.0/). 

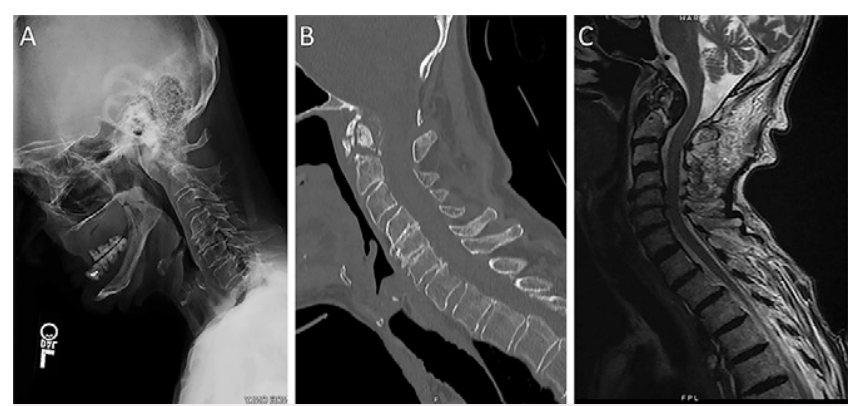

FIG. 1. Preoperative radiograph (A), CT scan (B), and T2-weighted magnetic resonance image $(\mathbf{C})$ of type 2 odontoid fracture with posterior displacement of dens.

To the best of our knowledge, 7D's MvIGS technology is the only navigation system that allows for real-time intraoperative navigation guidance with a CTA, and this is the first reported case using the technology in a $\mathrm{C} 1-2$ posterior fusion.

\section{Illustrative Case}

\section{History and Presentation}

An 86-year-old woman with severe osteoporosis (T-score -6.3), hypertension, and depression suffered a type 2 displaced odontoid fracture after a ground-level fall that was conservatively managed with an Aspen collar at an outside institution. She presented to our clinic several months after the fall with persistent axial neck pain, intermittent numbness in both hands, and unsteady gait. Her neurological exam was normal without evidence of myelopathy. Magnetic resonance imaging of the patient's cervical spine demonstrated a type 2 odontoid fracture with posterior displacement of the fractured dens and evidence of nonunion (Fig. 1). Given the failure of bony fusion with conservative therapy and the encroachment of the displaced odontoid fracture into the canal, surgical intervention was recommended.

\section{Operative Technique}

The patient was positioned prone on a Jackson table with her head fixed in the radiolucent Mayfield frame. After subperiosteal exposure of the dorsal bony anatomy of the atlas and axis (with removal of all soft tissue), the MvIGS system was brought into the field, and the clamp was placed on the $\mathrm{C} 2$ spinous process. Flash registration was performed, matching 1924 points from the $\mathrm{C} 1$ posterior arch and 1877 points from the $\mathrm{C} 2$ lamina and pars onto the preoperative CTA (Fig. 2A, B, D, and E). The entire registration process took 171 seconds ( $<3 \mathrm{~min}$ ) and allowed us to navigate our screw placement based on $3 \mathrm{D}$ reconstructions of the cervical spine that included the vascular anatomy (Fig. 2C, F, and G). First, we used the navigated probe (Fig. 3A) to determine the screw trajectory, which was started with the high-speed air drill. Next, we used the navigated drill guide (Fig. 3B) to drill the screw trajectory and the navigated tap (Fig. $3 \mathrm{C}$ ) to tap the trajectory. The screws were placed using the virtual K-wire feature of the MvIGS system (Fig. 3D and E). All screws accurately followed the trajectory mapped by the MvIGS system, as indicated in the figure with the planned trajectories superimposed on the final screw placement seen in the intraoperative 0 -arm spin (Fig. 4). A fibular strut graft was also placed in the C1-2 space and secured via a wiring technique to encourage bony fusion. An intraoperative CT scan was obtained to confirm accurate screw placement, per the surgeon's standard protocol.

\section{Postoperative Course}

The patient remained neurologically intact and was discharged to a skilled nursing facility after an uncomplicated hospital course. At her 6-week and 3-month postoperative visits, the patient reported improving incisional neck pain and walked well with a walker. Postoperative radiographs demonstrated excellent hardware position (Fig. 5).

\section{Discussion}

\section{Observations}

Atlantoaxial fixation is challenging because of the complex regional anatomy, the small size of the C1 lateral masses and C2 pedicles, and the proximity of important neurovascular structures, including the vertebral arteries. ${ }^{11-13}$ The incidence of anomalous vertebral arteries ranges from $2.7 \%$ in cadaveric studies to $5.4 \%$ in imaging studies. ${ }^{14,15}$ Although the incidence of vertebral artery
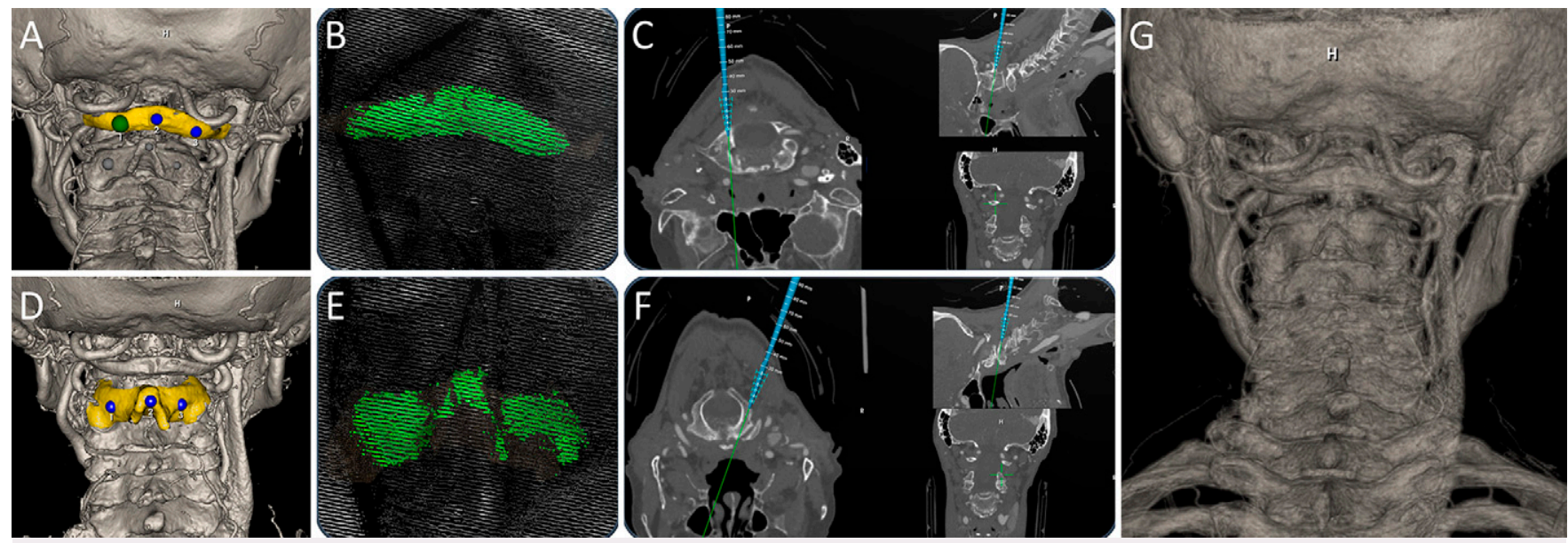

FIG. 2. MvIGS system. Bony landmarks on $C 1$ and $C 2$ were selected (A and $D)$, and registration of $C 1(B)$ and $C 2(E)$ vertebrae was performed. A navigated probe was used to guide screw trajectory $(\mathbf{C}$ and $\mathbf{F})$ on a $3 \mathrm{D}$ reconstruction of vertebral artery anatomy $(\mathbf{G})$. 

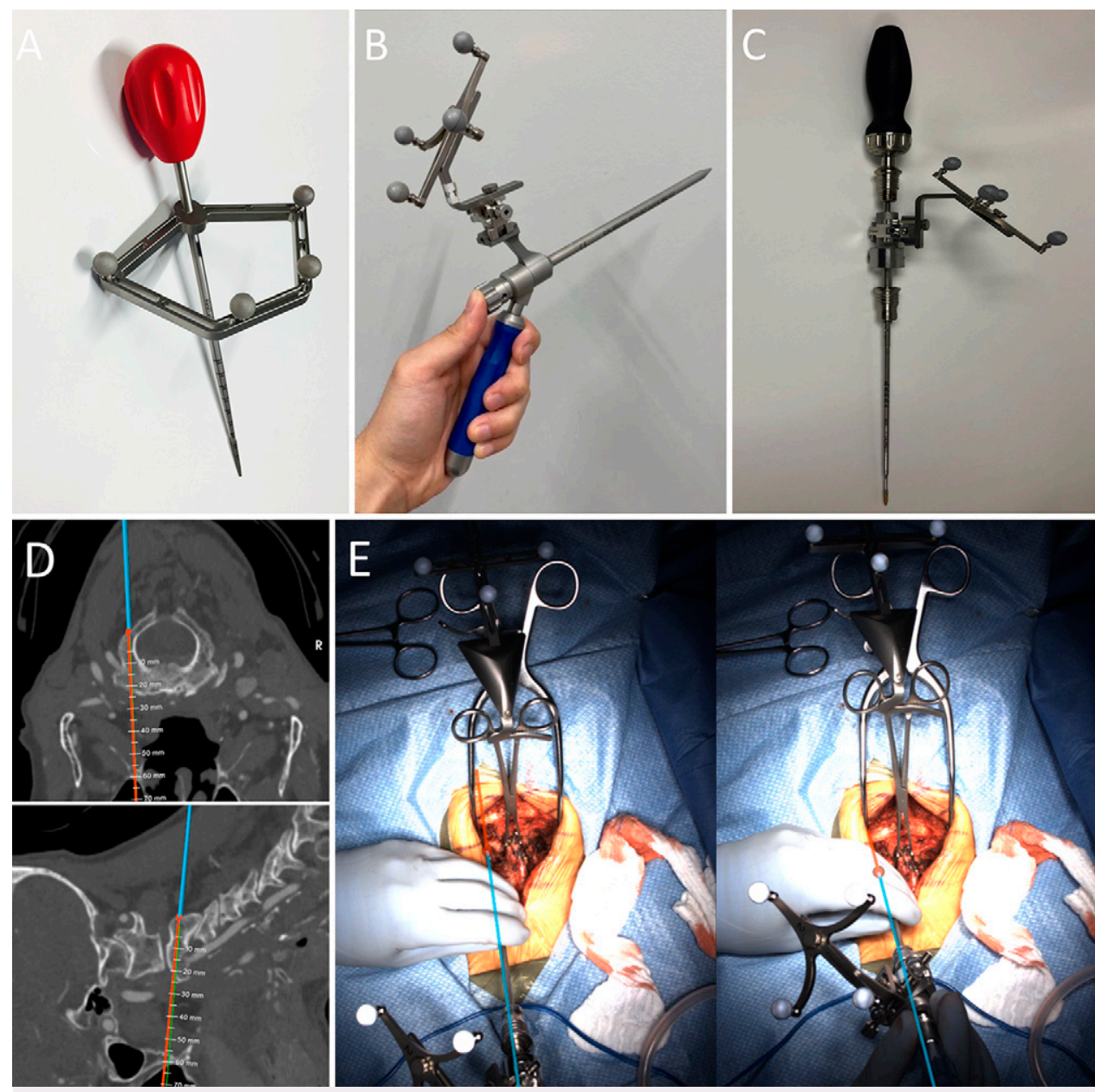

FIG. 3. Navigated screw placement. A navigated cervical pedicle probe (A), drill guide with universal tracker (B), and tap with universal tracker (C) were used to drill and tap screw trajectory. The virtual K-wire feature (D and E) was used to guide screw placement.
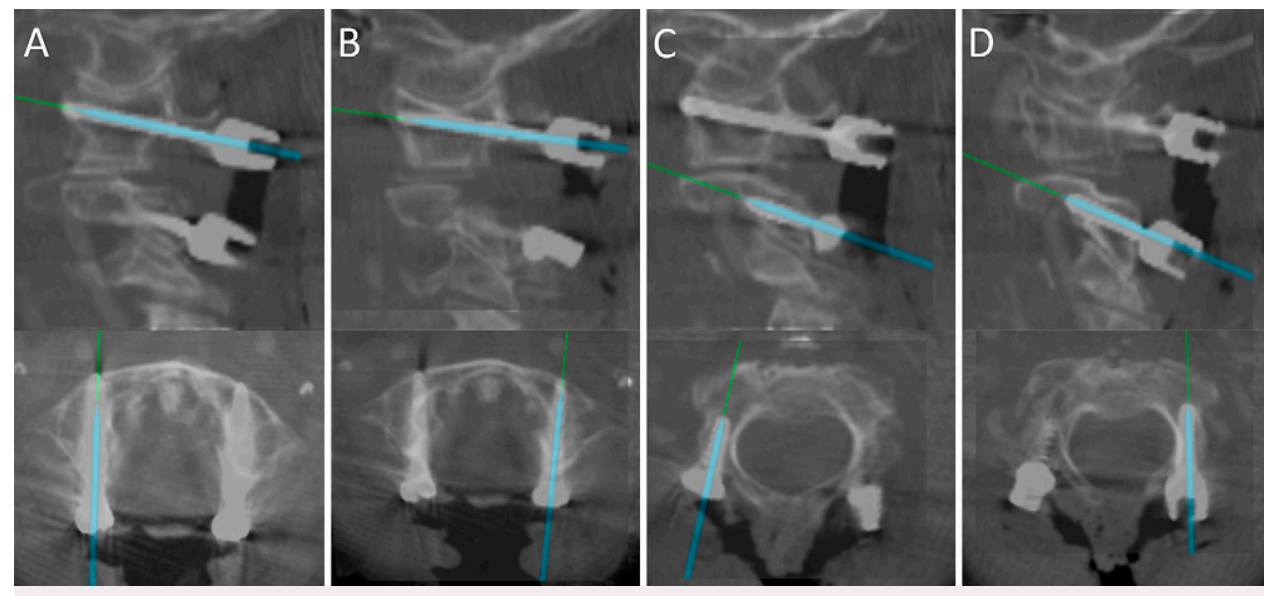

FIG. 4. Screw trajectory alignment. Pedicle screws were placed into the $C 1$ ( $A$ and $B$ ) and $C 2$ ( $C$ and $D$ ) vertebrae along the trajectories mapped by the MvIGS system. 

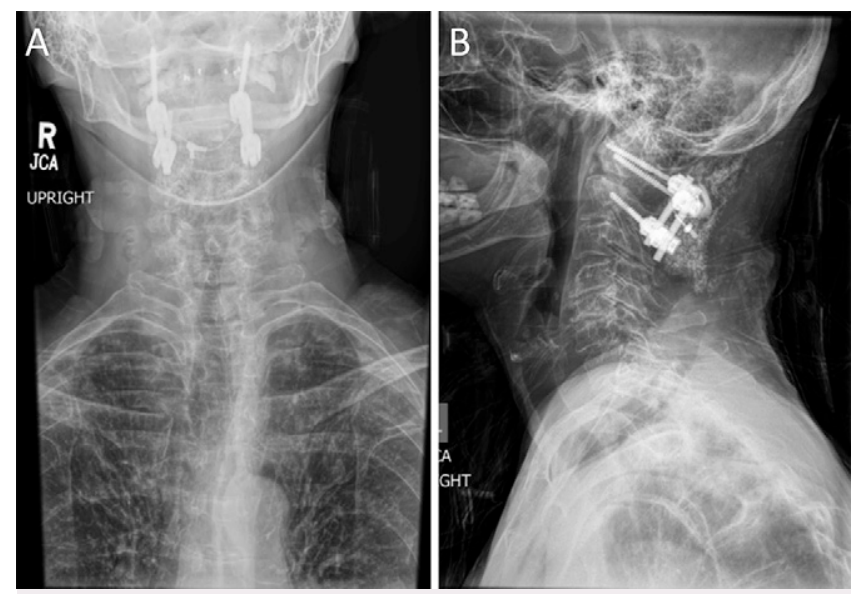

FIG. 5. Six-week postoperative posteroanterior (A) and lateral (B) radiographs demonstrating stable hardware.

injury is low, such injuries can be devastating and lead to permanent neurological injury, stroke, or death in $10 \%$ to $20 \%$ of cases. ${ }^{16,17}$ Although previous navigation systems enabled the use of a preoperative CT scan for intraoperative real-time navigation, ${ }^{18}$ angiograms had not yet been used for this purpose. To the best of our knowledge, this study is the first to demonstrate the feasibility of real-time intraoperative navigation with MvIGS using a preoperative CTA.

As discussed previously, MvIGS system technology registers onto a preoperative CT scan or CTA in the span of seconds to a few minutes. ${ }^{19}$ In addition, if the patient or the reference clamp moves during the procedure, the system can be readjusted rapidly with a repeat Flash Fix registration that takes seconds, although this feature was not used in our case. For $\mathrm{C} 1-2$ posterior fusions in particular, moving the spinous process clamp can be helpful between screw placements when the clamp is in the way of a specific screw trajectory. This technique has been used by the senior author (C.C.Z.) in other cases of C1-2 instrumentation. Also of note, the MvIGS system is a navigation platform only and can be used with spine implants from any company.

\section{Lessons}

Yamazaki and colleagues have demonstrated the value of preoperative 3D CTA reconstructions for identifying abnormal vertebral artery anatomy. ${ }^{20}$ Although CT-guided navigation is becoming the standard of care in spine surgery, ${ }^{21,22}$ with improved screw accuracy and decreased fluoroscopic time and blood loss compared to those with traditional fluoroscopic and free-hand techniques, ${ }^{23}$ traditional spinal navigation systems are unable to visualize vascular anatomy. Hsu et al. found that $50 \%$ of cases of vertebral artery injury revealed anomalous vessel anatomy during postoperative review, ${ }^{24}$ which highlights the importance of preoperative and, potentially, intraoperative visualization of vertebral vasculature. MvIGS offers the additional benefit of intraoperative navigation using a 3D CTA reconstruction, which may enhance surgeon screw placement accuracy and avoid vascular complications. Although navigation systems, including those by 7D, require additional upfront setup time, operating room efficiency may improve in the long term as surgeons and their operative teams overcome the learning curve. ${ }^{25}$

We present a case of CTA-guided navigation during C1-2 posterior fusion for type 2 odontoid fracture using MvIGS. The MvIGS system minimizes workflow disruption and does not increase radiation exposure. 3D reconstruction of the regional bony and vascular anatomy assists surgeons during screw placement. CTA-guided navigation offers promise in assisting surgeons during complex craniocervical cases.

\section{References}

1. Hong JT, Lee SW, Son BC, et al. Analysis of anatomical variations of bone and vascular structures around the posterior atlantal arch using three-dimensional computed tomography angiography. J Neurosurg Spine. 2008;8(3):230-236.

2. Yeom JS, Buchowski JM, Kim HJ, et al. Risk of vertebral artery injury: comparison between $\mathrm{C} 1-\mathrm{C} 2$ transarticular and $\mathrm{C} 2$ pedicle screws. Spine J. 2013;13(7):775-785.

3. Wright NM, Lauryssen $\mathrm{C}$. Vertebral artery injury in $\mathrm{C} 1-2$ transarticular screw fixation: results of a survey of the AANS/CNS section on disorders of the spine and peripheral nerves. J Neurosurg. 1998;88 (4):634-640

4. Madawi AA, Casey ATH, Solanki GA, et al. Radiological and anatomical evaluation of the atlantoaxial transarticular screw fixation technique. J Neurosurg. 1997;86(6):961-968.

5. Burke JP, Gerszten PC, Welch WC. latrogenic vertebral artery injury during anterior cervical spine surgery. Spine J. 2005;5(5):508-514.

6. Daentzer D, Deinsberger W, Böker DK. Vertebral artery complications in anterior approaches to the cervical spine: report of two cases and review of literature. Surg Neurol. 2003;59(4):300-309.

7. Du JP, Fan Y, Wu QN, et al. Accuracy of pedicle screw insertion among 3 image-guided navigation systems: systematic review and meta-analysis. World Neurosurg. 2018;109:24-30.

8. Mason A, Paulsen R, Babuska JM, et al. The accuracy of pedicle screw placement using intraoperative image guidance systems. $J$ Neurosurg Spine. 2014;20(2):196-203.

9. Lee $\mathrm{CH}$, Hong JT, Kang DH, et al. Epidemiology of iatrogenic vertebral artery injury in cervical spine surgery: 21 multicenter studies. World Neurosurg. 2019;126:e1050-e1054.

10. Faraji-Dana Z, Mariampillai ALD, Standish BA, et al. Machine-vision Image-Guided Surgery for spinal and cranial procedures. In: Abedin-Nasab MH, ed. Handbook of Robotic and Image-Guided Surgery. Elsevier; 2020:551-574.

11. Lall R, Patel NJ, Resnick DK. A review of complications associated with craniocervical fusion surgery. Neurosurgery. 2010;67(5):13961403.

12. Neo M, Sakamoto T, Fujibayashi S, Nakamura T. The clinical risk of vertebral artery injury from cervical pedicle screws inserted in degenerative vertebrae. Spine (Phila Pa 1976). 2005;30(24):28002805.

13. Yeom JS, Buchowski JM, Park KW, et al. Undetected vertebral artery groove and foramen violations during $\mathrm{C} 1$ lateral mass and $\mathrm{C} 2$ pedicle screw placement. Spine (Phila Pa 1976). 2008;33(25): E942-E949.

14. Curylo LJ, Mason HC, Bohlman HH, Yoo JU. Tortuous course of the vertebral artery and anterior cervical decompression: a cadaveric and clinical case study. Spine (Phila Pa 1976). 2000;25 (22):2860-2864.

15. Hong JT, Park DK, Lee MJ, et al. Anatomical variations of the vertebral artery segment in the lower cervical spine: analysis by threedimensional computed tomography angiography. Spine (Phila $\mathrm{Pa}$ 1976). 2008;33(22):2422-2426.

16. Lunardini DJ, Eskander MS, Even JL, et al. Vertebral artery injuries in cervical spine surgery. Spine J. 2014;14(8):1520-1525. 
17. Burlew CC, Biffl WL. Imaging for blunt carotid and vertebral artery injuries. Surg Clin North Am. 2011;91(1):217-231.

18. Smith JD, Jack MM, Harn NR, et al. Screw placement accuracy and outcomes following 0-arm-navigated atlantoaxial fusion: a feasibility study. Global Spine J. 2016;6(4):344-349.

19. Nguyen NQ, Priola SM, Ramjist JM, et al. Machine vision augmented reality for pedicle screw insertion during spine surgery. $J$ Clin Neurosci. 2020;72:350-356.

20. Yamazaki M, Okawa A, Furuya T, et al. Anomalous vertebral arteries in the extra- and intraosseous regions of the craniovertebral junction visualized by 3-dimensional computed tomographic angiography: analysis of 100 consecutive surgical cases and review of the literature. Spine (Phila Pa 1976). 2012;37

(22):E1389-E1397.

21. Kim TT, Drazin D, Shweikeh F, et al. Clinical and radiographic outcomes of minimally invasive percutaneous pedicle screw placement with intraoperative CT (O-arm) image guidance navigation. Neurosurg Focus. 2014;36(3):E1.

22. Singh PK, Garg K, Sawarkar D, et al. Computed tomography-guided C2 pedicle screw placement for treatment of unstable hangman fractures. Spine (Phila Pa 1976). 2014;39(18):E1058-E1065.

23. Yang YL, Zhou DS, He JL. Comparison of isocentric C-arm 3-dimensional navigation and conventional fluoroscopy for $\mathrm{C} 1$ lateral mass and C2 pedicle screw placement for atlantoaxial instability. $J$ Spinal Disord Tech. 2013;26(3):127-134.

24. Hsu WK, Kannan A, Mai HT, et al. Epidemiology and outcomes of vertebral artery injury in 16582 cervical spine surgery patients: an AOSpine North America multicenter study. Global Spine J. 2017;7 (1 suppl):21S-27S.
25. Khanna AR, Yanamadala V, Coumans JV. Effect of intraoperative navigation on operative time in 1-level lumbar fusion surgery. J Clin Neurosci. 2016;32:72-76.

\section{Disclosures}

Dr. Zygourakis is a consultant for Stryker, SpineAlign, 7D, and Globus. Dr. Zygourakis also reported personal fees from 7D while conducting the study and personal fees from Stryker outside the submitted work.

\section{Author Contributions}

Conception and design: Zygourakis, Medress, Leung, Sklar. Acquisition of data: Zygourakis, Wadhwa. Analysis and interpretation of data: Zygourakis, Wadhwa, Medress, Leung, Sklar. Drafting the article: Wadhwa, Malacon, Medress. Critically revising the article: all authors. Reviewed submitted version of manuscript: Zygourakis, Wadhwa, Malacon, Medress, Sklar. Approved the final version of the manuscript on behalf of all authors: Zygourakis. Administrative/ technical/material support: Zygourakis. Study supervision: Zygourakis, Medress.

\section{Correspondence}

Corinna C. Zygourakis: Stanford University School of Medicine, Palo Alto, CA. corinnaz@stanford.edu. 